\title{
111. Experimentelle Erzeugung des Sarkoms bei weissen Ratten durch kombinierte subkutane Applikation von Kieselgur und o-Aminoazotoluol.
}

\author{
Von Takaoki SASAKI, Naoaki NAGAo und Ichiro OTSUKA. \\ Sasaki-Laboratorium, Tokyo.
}

(Comm. by T. SASAKI, M.I.A., Nov, 12, 1940.)

Vor der Anstellung der Fütterungsversuche mit o-Aminoazotoluol von Sasaki und Yoshida (Proc. 8 (1932), 464), durch welche die carcinogene Wirkung des o-Aminoazotoluols auf Rattenleber festgestellt worden war, waren auch die Injektionsversuche mit derselben Substanz bei Ratten ausgeführt worden (Yoshida, Gann 28, 441, 1934). Dabei wurde zwar Leberkrebs beobachtet, aber auch Hautcarcinom bei 2 Fällen konstatiert. Das Carcinom entstand nicht in der Injektionsstelle, sondern in einer ganz entfernten Stelle. Cysticercussarkom wurde auch dabei überaus häufig beobachtet.

Y. Nishiyama in hiesiegem Laboratorium erzeugte bei Ratten Sarkome in loco durch wiederholte subkutane Injektionen von konzentrierter Glukoselösung. Dabei entstand Sarkom bei den gleichzeitig mit oAminoazotoluol gefütterten Fällen viel häufiger als bei den nicht gefütterten Fällen. Somit scheint o-Aminoazotoluol nicht nur Leberkrebs hervorzubringen imstande zu sein, sondern auch eine begünstigende Wirkung auf Sarkombildung zu haben (Gann 32, 85, 1938). Tanaka konnte auch ein Reticulosarkom durch Injektion des 4-N-Dimethylaminoazobenzols (Strukturisomer des o-Aminoazotoluols) bei Ratten erzeugen (Gann 32, 275, 1938, Osaka .Ig. Z. 36, 1774, 1937). Bei den ausgedehnten Versuchen von englischen sowie amerikanischen Forschern mit zahlreichen polyzyklischen aromatischen carcinogenen Substanzen wurde bei der Hautbepinselung der Substanz hauptsächlich Carcinom und bei der subkutanen Injektion Sarkom konstatiert. Somit scheint zwischen der Carcinom- und Sarkombildung ein gewissermassen gemeinsamer tumorbildender (carcinogen im weiteren Sinne) Faktor zu vorhanden.

Andererseits beschäftigte Podwyssotzki sich im Jahre 1912 mit der experimentellen Erzeugung der Riesenzellengranulomen durch Kieselgur. Er benutzte zu seinem Versuche eine Aufschwemmung von Kieselgur in physiologischer Kochsalzlösung und injizierte sie dem Meerschweinchen intraperitoneal. Der dabei entstandene Knoten bestand aus dicht aneinander liegenden kolossal grossen plasmodien- oder syncytienähnlichen mit zahlreichen Kernen besetzten Protoplasmassen oder Riesenzellen (Zieglers Beitr. 47, 270, 1910). Schirokogoroff prüfte dieses Experiment nach und benannte ihn den dem Riesenzellensarkom äusserst ähnlichen gutartigen Tumor (Virchows Arch. 205, 166, 1911). Mit diesem Riesenzellengranulome stellte Stieve Transplantationsversuche an. Aus 9 Fällen wurde das Granulom 50 Meerschweinchen übergepflanzt. Nur bei einem Fall aus einem Stamme zeigte das Transplantat ein selbständiges Wachstum. Dabei zeigte das Transplantat viele Mitosen- 
figuren und wuchs in die Darmwand infiltrierend hinein, enthielt aber noch Kieselgur in sich (Zieglers Beitr. 54, 415, 1912). Morphologische Veränderungen von Kieselgurgranulomen unter dem Einfluss des Bazillenstammes Hü (Pickham, Z. Krebsf. 23, 496, 1926) und auch der Einfluss der Prolan-injektion auf Kieselgurgranulom (Caccia, Tumori 10, 38, 1936, ref. in Am. J. Canc. 32, 463, 1938) wurden auch untersucht. Eine echte Geschwulst wurde aber dabei nicht erzeugt.

Auf Grund der obengenannten Tatsachen wurde in hiesigem Laboratorium der Kombinationsversuch von o-Aminoazotoluol und Kieselgur studiert. Die Kieselgursuspension in Olivenöl wurde den Ratten subkutan mehrmals injiziert. Dabei wurde o-Aminoazotoluol gleichzeitig einerseits subkutan andererseits per os einverleibt. Bei 6 Fällen entstand Sarkom in loco und ein davon war übertragbar. Histologisch war es spindelzelliges oder polymorphzelliges Sarkom und enthielt weder mehrkernige Fremdkörperriesenzellen noch Kieselgurreste.

Somit wurde festgestellt, dass o-Aminoazotoluol unter Umständen Sarkombildung begünstigt, wenn auch nicht so stark wie carcinogene polyzyklische Kohlenwasserstoffe. Die Frage, ob es sich dabei um die Steigerung der allgemeinen Tumorbereitschaft oder nur um eine lokale Wirkung der Substanz handelt, bleibt noch unentschieden.

\section{Tierversuche.}

Kieselgur ,, Kahlbaum “ wurde zuerst mit verdünnter Salzsäure gekocht, mit Wasser ausgewaschen, im Achatmörser fein pulverisiert und gesiebt. Diese ganz feine Kieselgur wurde im Verhältnis von $1 \mathrm{~g} \mathrm{zu}$ $10 \mathrm{ccm}$ in Olivenöl oder im 1\% o-Aminoazotoluol enthaltenden Olivenöl suspendiert.

Bei der ersten Versuchsreihe wurden 30 Ratten die o-Aminoazotoluol enthaltenden Kieselgursuspension je $0.5 \mathrm{ccm}$ oder $1.0 \mathrm{ccm}$ wöchentlich einmal subkutan in dieselbe Stelle injiziert. Die Tiere wurden mit unpolierten Reikörnern, die o-Aminoazotoluol nicht enthielt, ernährt. Bei der zweiten Versuchsreihe wurden 20 Ratten die Kieselgursuspension, die o-Aminoazotoluol nicht enthielt, in derselben Weise injiziert. Die Tiere wurden aber dabei mit dem $0.1 \%$ o-Aminoazotoluol enthaltenden Futter ernährt. Bei der dritten Versuchsreihe wurden schliesslich 10 Ratten mit der o-Aminoazotoluol nicht enthaltenden Kieselgursuspension injiziert und gleichzeitig mit dem o-Aminoazotoluol auch nicht enthaltenden Reisfutter ernährt.

Nach etwa 12 maligen Injektionen wurde die weitere Injektion unterbrochen. Nach etwa 100 oder 180 tägiger Fütterung mit dem o-Aminoazotoluol enthaltenden Reisfutter wurde das Futter in das gewöhnliche o-Aminoazotoluol nicht enthaltende Futter umgesetzt.

Bei allen drei Versuchsreihen entstand in loco nach den 12 maligen Injektionen eine Konglomeratbildung, die aus miteinander fest verwachsenen von linsen- bis erbsengrossen knorpelharten Knoten bestand und von der Umgebung scharf begrenzt war. Dieses Knoten blieb bei den meisten Fällen ziemlich unverändert und weder vergrösserte noch verkleinerte selbst nach einjähriger Versuchsdauer. 
Das Knoten war gelblich grau, knorpelhart und schwer schneidbar. Seine Schnittfläche war fast gleichmässig, gelblich und sah etwas abgetrocknet aus. Histologisch war der zentrale Teil ganz kernarm und bestand aus einer Durchflechtung grober, hyalinisierter Bündeln der kollagenen Fasern und dazwischen liegenden Kieselgurpartikelchen. Das Kieselgurpartikelchen war selbst nach einer langen Versuchsdauer mikroskopisch sehr leicht zu erkennen. Im peripheren Teil befand sich eine Zone von einem schmalen Granulatonsgewebe, welches eine Neubildung von zarten kollagenen Fasern begleitete und auch Kieselgurpartikelchen und mehrkernige Fremdkörperriesenzellen enthielt.

Bei 4 Fällen von der ersten Versuchsreihe (add. o-Aminoazotoluol subkutan) und bei 2 Fällen von der zweiten Versuchsreihe (add. oAminoazotoluol per os) fing dann ziemlich plötzlich ein Knoten in dem oben erwähnten Konglomerat ein schnelles Wachstum zu zeigen an. Das Knoten entwickelte sich in 2 bis 4 Wochen zu einem Tumor von der Grösse einer Haselnuss oder Walnuss. Der Tumor war von grauweisser Farbe, elastisch und von weniger derber Konsistenz als andere Konglomeratknoten. Er war leicht schneidbar und seine Schnittfläche markig, grauweiss und feucht. Histologisch zeigte der Tumor das Bild des Spindelzellensarkoms oder polymorphzelligen Sarkoms. Bei einem Fall von polymorphzelligen Sarkom wuchsen die Tumorzellen infiltrierend in das Muskelgewebe hinein.

Das Kieselgurpartikelchen, das immer im Konglomeratknoten nachgewiesen wurde, wurde im Sarkomgewebe nicht gefunden, mit Ausnahme eines einzigen Falls. Das Spindelzellensarkom war oft von einer Neubildung der zarten kollagenen Fasern begleitet und zeigte eine fibroplastische Natur. Das Sarkomgewebe wurde bis zur vierten Generation mit Erfolg transplantiert.

Zwischen dem Sarkom und dem Granulationsgewebe des Konglomeratknotens liess sich ein Übergangsbild erkennen. Das schmale Granulationsgewebe verbreitete sich an verschiedenen Stellen, oft sogar im zentralen Teil des Knotens. In diesem Granulationsgewebe nahmen die Wander- und Fremdkörperriesenzellen an Zahl ab. Die Fibroblasten waren dabei meistens gleichmässig gestaltet, zuweilen aber zeigten sie eine gewisse Zellatypie. Plump verdickten oder unregelmässig gestaltete Kernen oder Mitosenfiguren traten dabei oft auf. Das Kieselgurpartikelchen war dabei noch leicht zu finden.

Bei der dritten Versuchsreihe (d. i. Kieselgur allein ohne o-Aminoazotoluolzugabe) wurde keine Sarkombildung selbst nach sehr langer Versuchsdauer beobachtet.

Die ausführliche Mitteilung wird demnächst in ,Gann “ erscheinen. 
TABELLE.

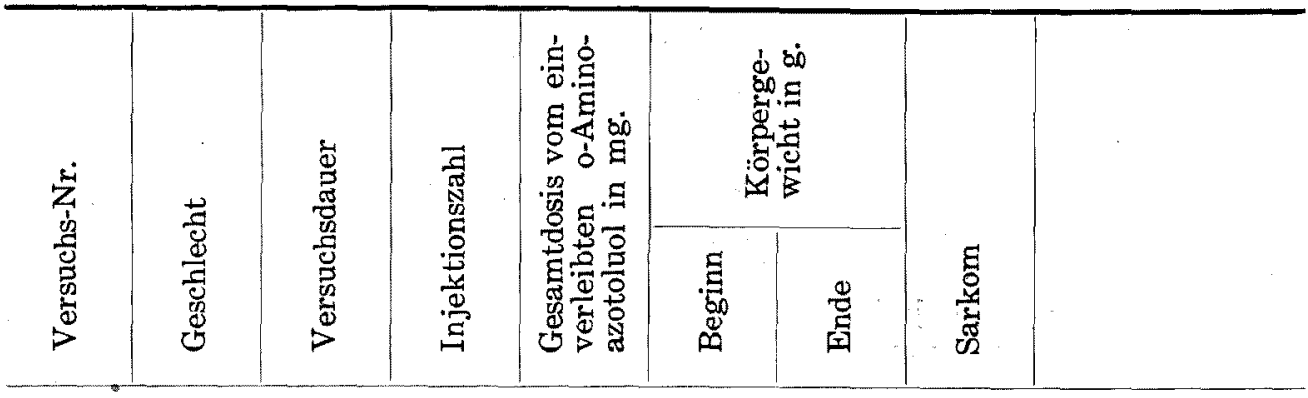

30 Versuchstiere, mit der o-Aminoazotoluol enthaltenden Kieselgursuspension injiziert, mit dem o-Aminoazotoluol nicht enthaltenden Futter ernährt, darunter innerbalb 326 Tagen getöteten Fälle aus der Tabelle ausgelassen.

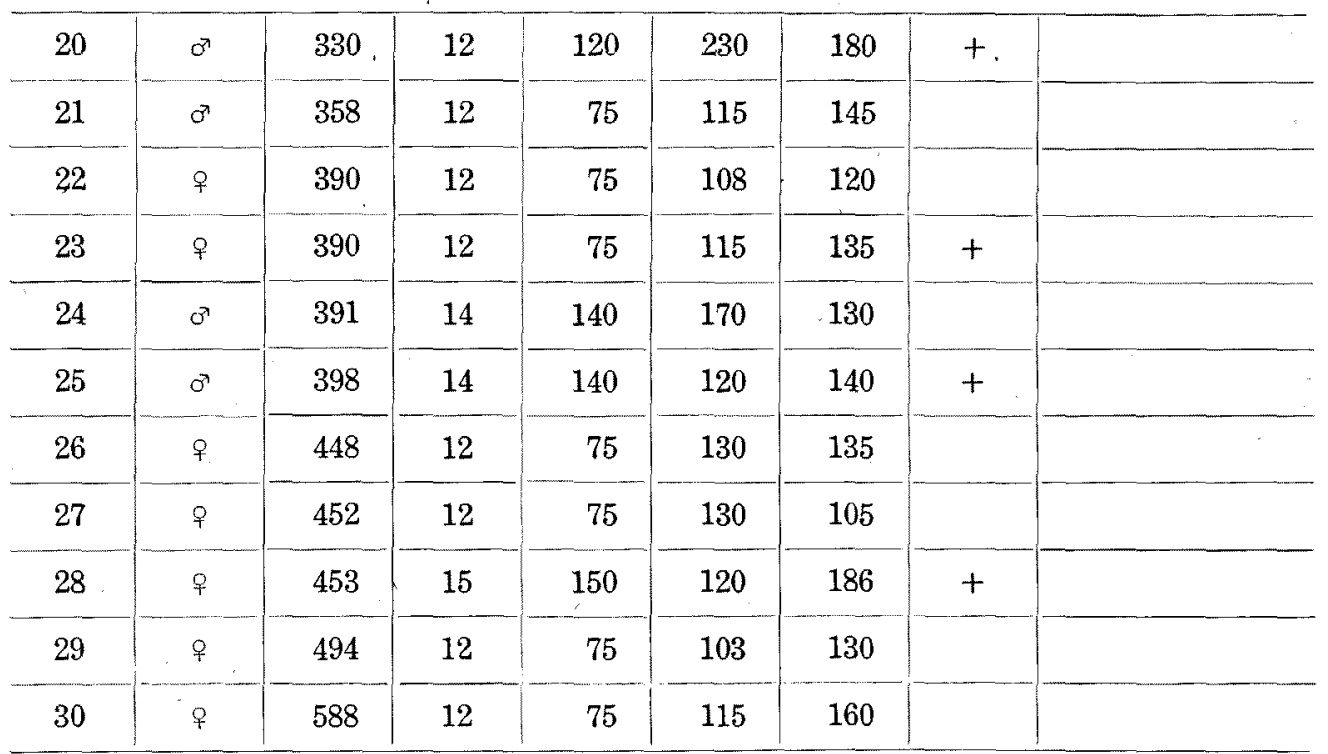

20 Versuchstiere, mit der o-Aminoazotoluol nicht enthaltenden Kieselgursuspension injiziert, mit dem o-Aminoazotoluol enthaltenden Futter ernährt, darunter innerhalb 400 Tagen getöteten Fälle aus der Tabelle ausgelassen.

\begin{tabular}{l|c|c|c|c|c|c|c|c}
\hline 13 & $\sigma^{7}$ & 404 & 19 & 940 & 200 & 100 & \\
\hline 14 & 0 & 411 & 12 & 1550 & 105 & 120 & & \\
\hline 15 & 0 & 425 & 12 & 1650 & 125 & 110 & \\
\hline 16 & 0 & 476 & 12 & 1700 & 130 & 205 & + \\
\hline 17 & 9 & 483 & 12 & 1650 & 115 & 125 & \\
\hline 18 & 9 & 499 & 14 & 850 & 155 & 90 & \\
\hline 19 & 9 & 509 & 12 & 1550 & 105 & 90 & \\
\hline 20 & 0 & 648 & 12 & 1550 & 115 & 170 & + \\
\hline
\end{tabular}


No. 9.] Experimentelle Erzeugung des Sarkoms bei weissen Ratten.

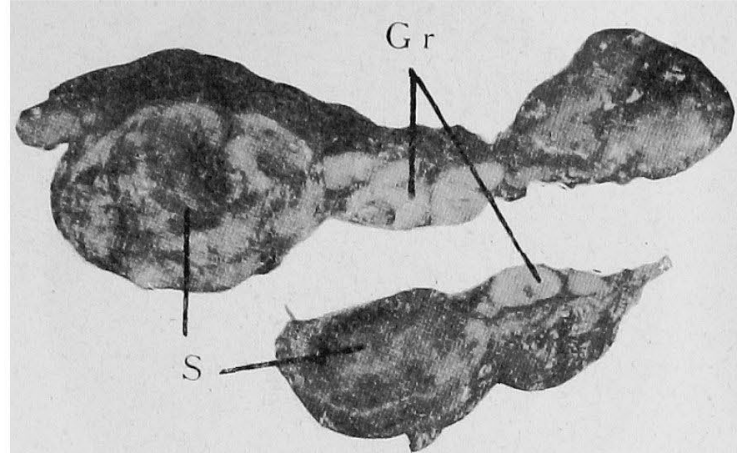

Abb. 1. (Nr. I-23; 390 Tage). $\mathrm{S}$-Sarkom, Gr-Kieselgurgranulom.

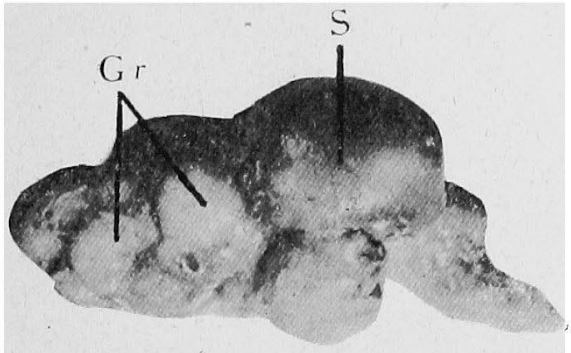

Abb. 2. (Nr. II-20; 648 Tage). S-Sarkom, Gr-Kieselgurgranulom.

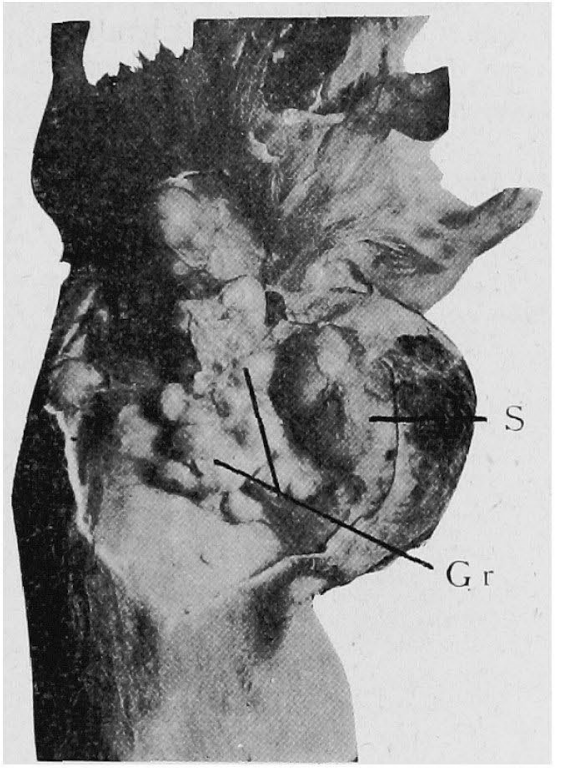

Abb. 3. (Nr. II-16; 476 Tage).

$\mathrm{S}-$ Sarkom, Gr-Kieselgurgranulom.

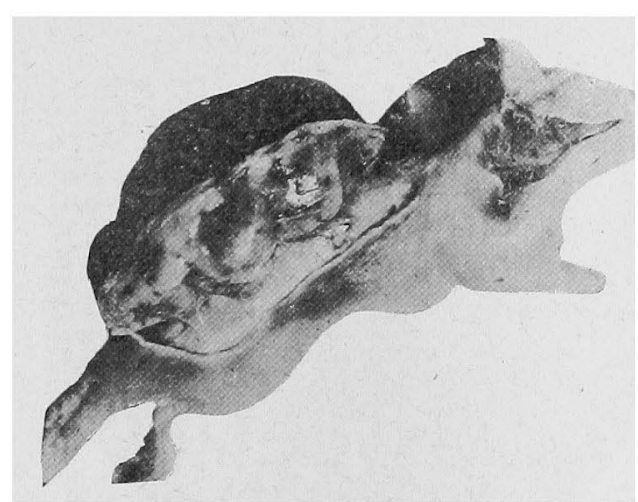

Abb. 5.

Subkutane Transplantation.

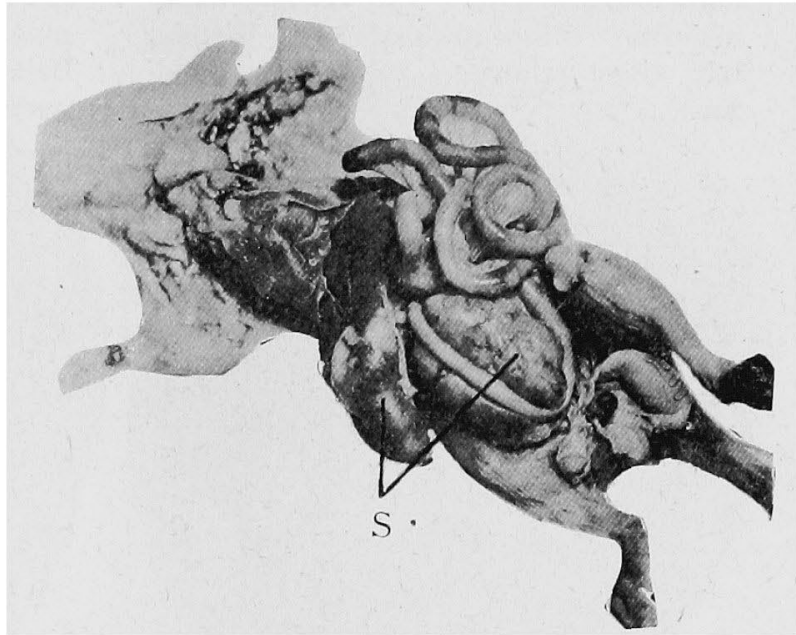

Abb. 4.

Intraperitoneale Transplanplantation. S-Transplantat.

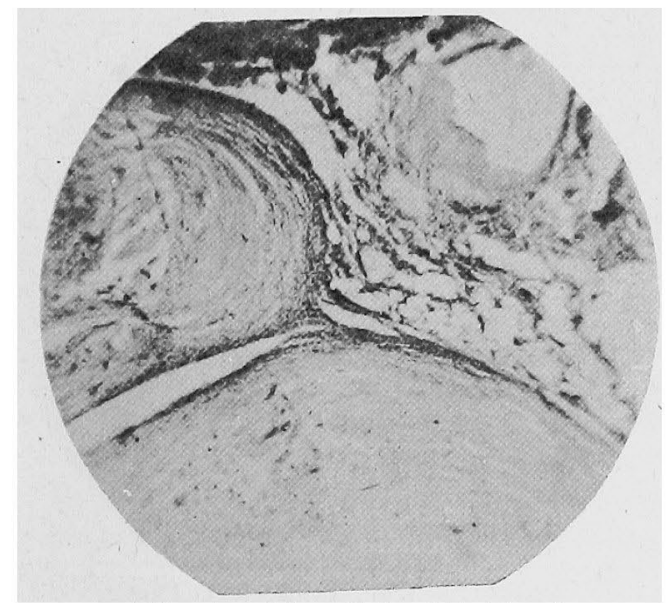

Abb. 6. (Nr. II-8; 340 Tage). Drei Kieselgurgranulomknoten, sich nebeneinander liegend. 


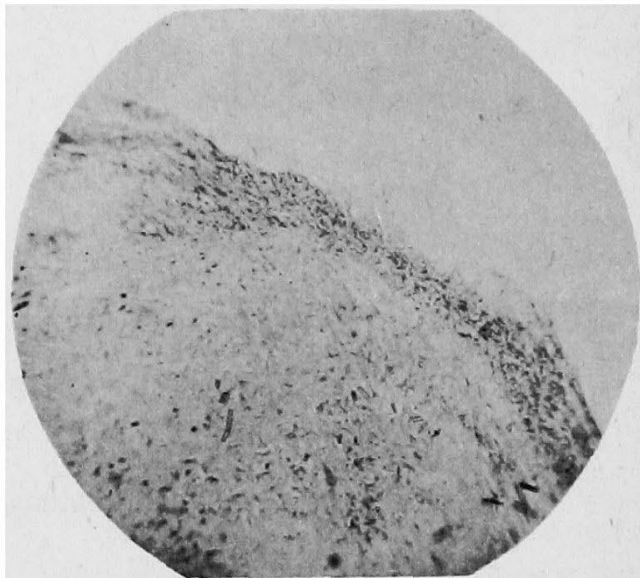

Abb. 7. (Nr. I-6; 295 Tage).

Kieselgurgranulom, aus groben hyalinisierten Bindegewebsfasern besteht, mit einer schmalen zellreichen Randzone.

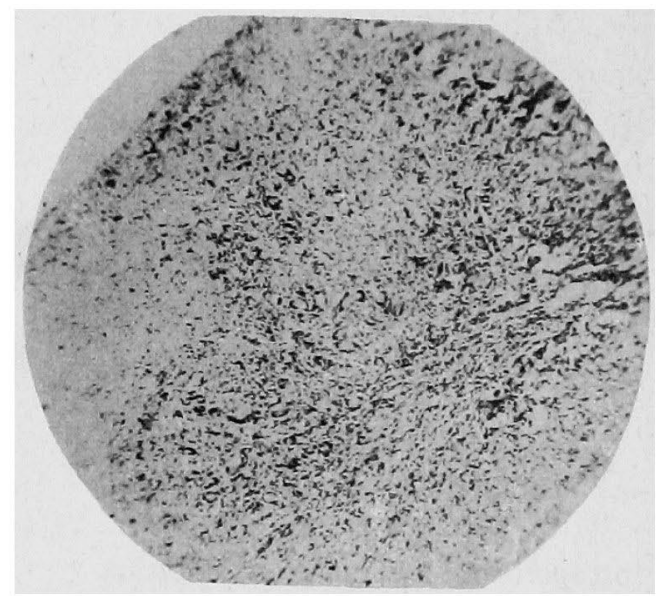

Abb. 9. (Nr. II-9; 363 Tage).

Randzone eines Kieselgurgranuloms, Zahlreiche mehrkernige Fremdkörperriesenzellen und dazwischen liegende Kieselgurpartikelchen.

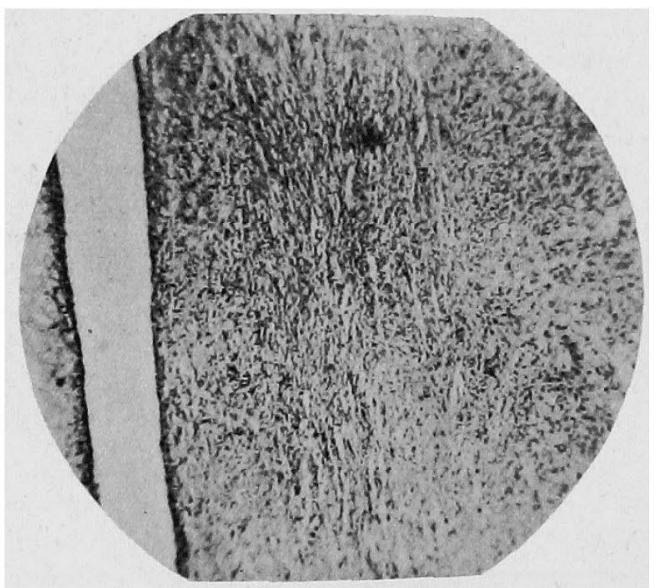

Abb. 11. (Nr. I-24; 391 Tage).

Randzone eines Kieselgurgranuloms, faserig, ohne Fremdkörperriesenzellen.

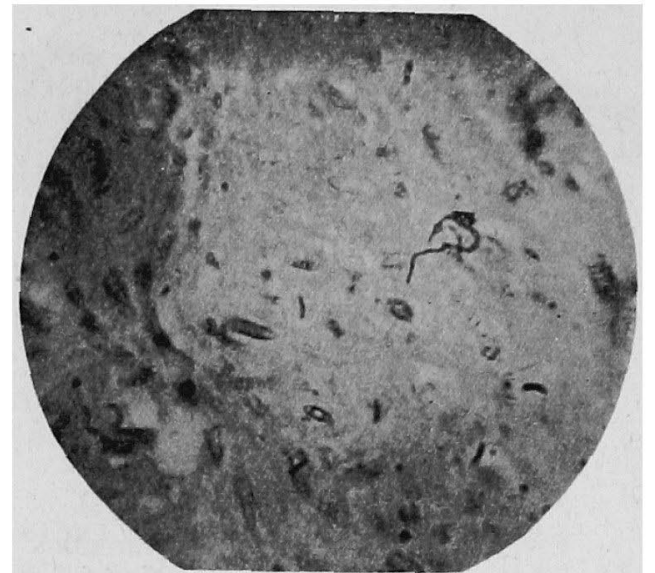

Abb. 8. (Nr. I-30; 588 Tage).

Zentrale Partie eines Kieselgurgranuloms, ganz kernarm, grobe hyaline Balken und dazwischen liegende Kieselgurpartikelchen.

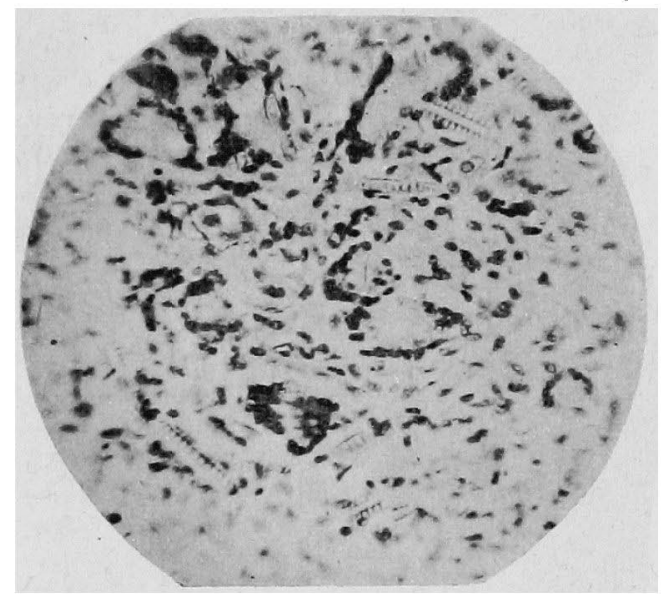

Abb. 10. (Nr. II-9; 363 Tage).

Dieselbe Präparatstelle von Abb. 9, stark vergrössert. Fremdköperriesenzellen und Keiselgurpartikelchen.

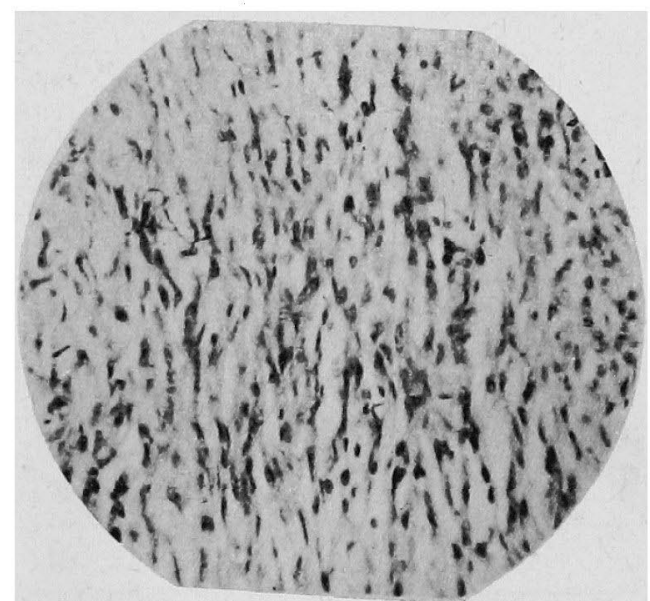

Abb. 12. (Nr. I-24; 391 Tage).

Dieselbe Präparatstelle von Abb. 11, stark vergrössert. Faseriges Granulationsgewebe mit Kieselgurpartikelchen. 


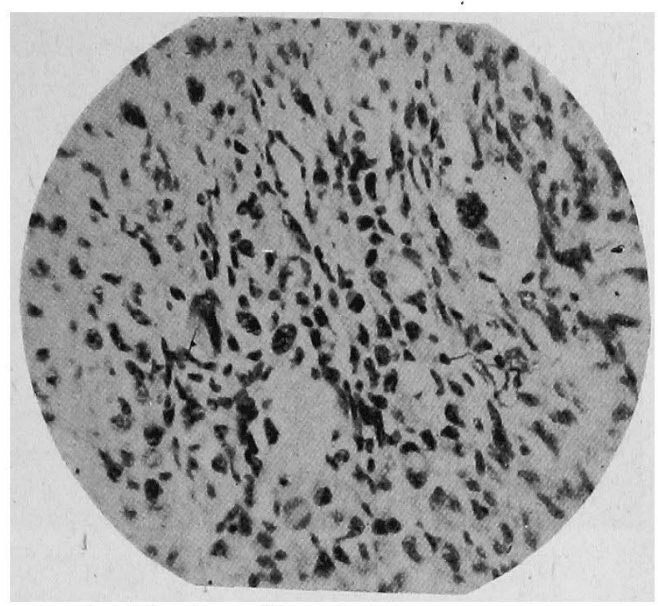

Abb. 13. (Nr. I-25; 398 Tage). Zellhaufen mit Zellatypie.

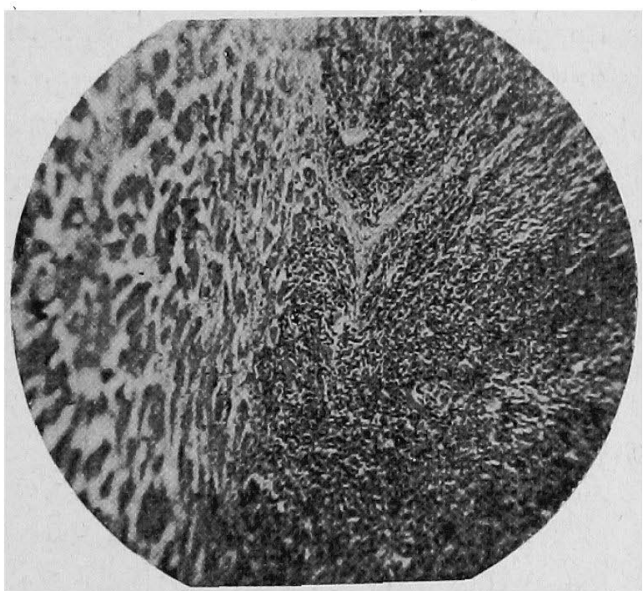

Abb. 15. (Nr. I-28; 453 Tage).

Infiltratives Wachstum des Sarkoms in Muskelfasern.

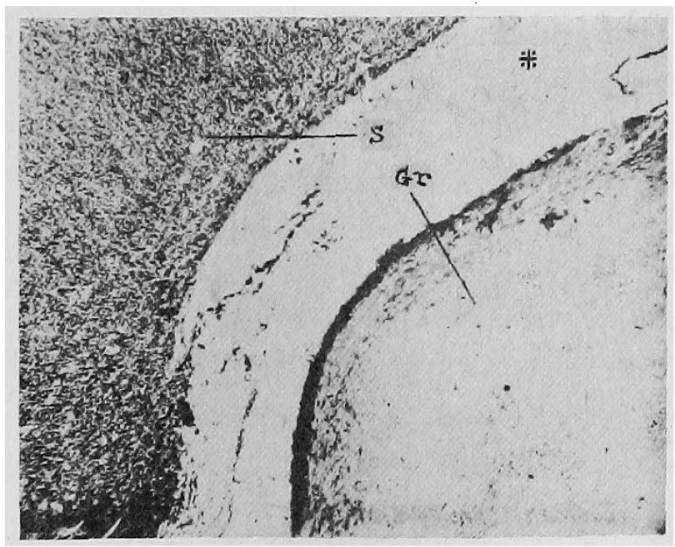

Abb. 17. (Nr. II-20; 648 Tage).

Mikroskopische Bild des Falls von Abb. 2. Kernarmes Kieselgurgranulom (Gr) und dicht darauf liegendes Sarkomgewebe (S).

*-Bei der Herstellung des Schnittes künstlich erzeugte Lücke.

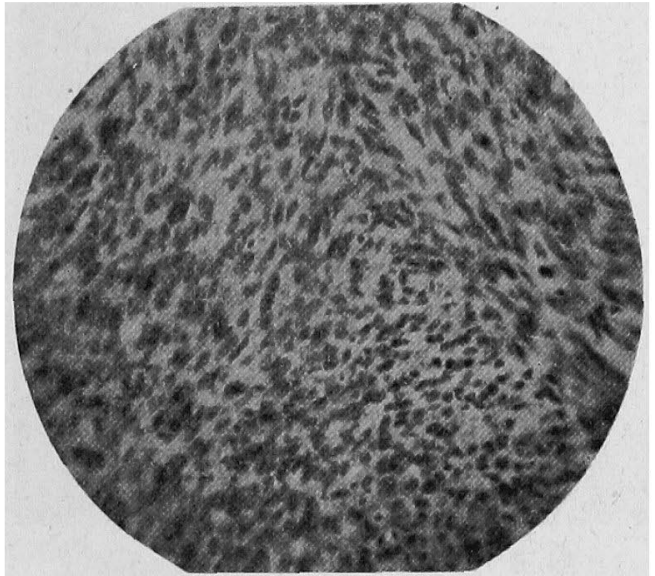

Abb. 14. (Nr. II-16; 476 Tage). Spindelzellensarkom.

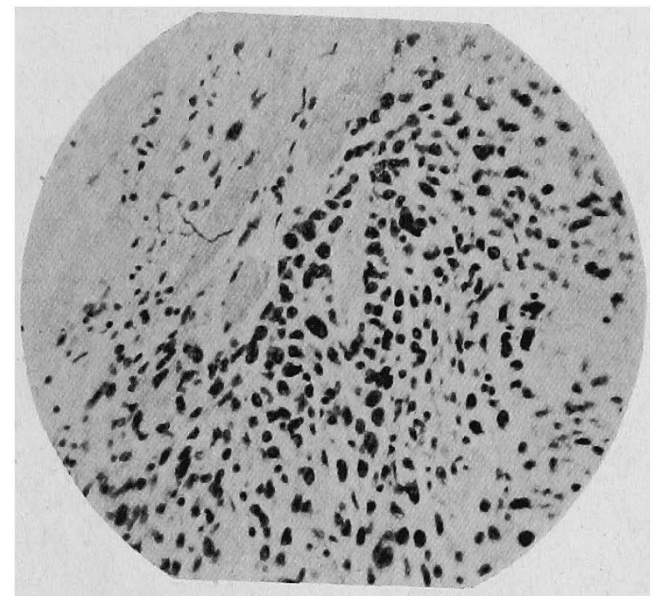

Abb. 16. (Nr. I-28; 453 Tage).

Dieselbe Präparatstelle von Abb. 15, stark vergrössert.

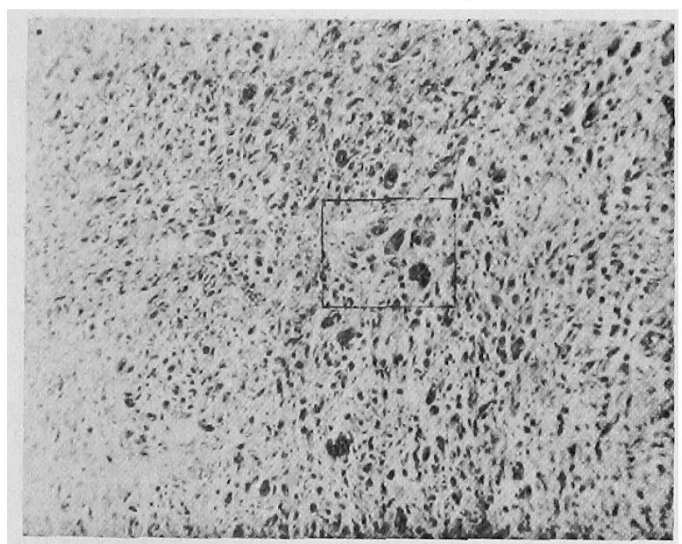

Abb. 18. (Nr. II-20; 648 Tage).

Derselbe Fall wie Abb. 17. Polymorphezelliges Sarkom. Mittelstark vergrössert. 


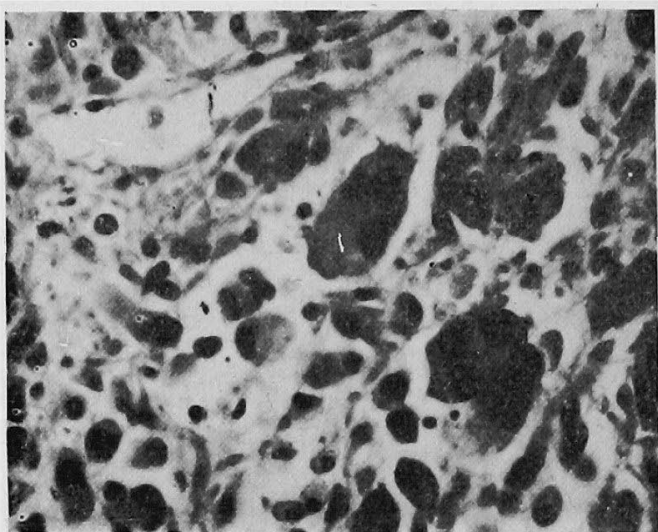

Abb. 19. (Nr. II-20; 648 Tage).

Derselbe Fall wie Abb. 17. Stark vergrössert.

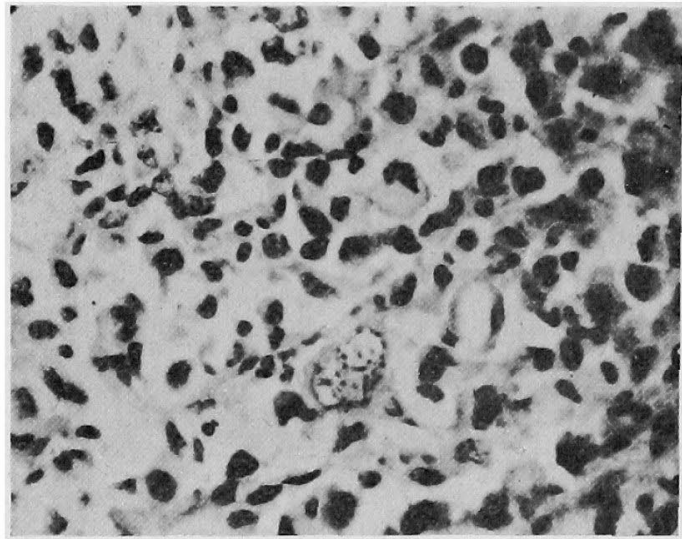

Abb. 21. (Nr. I-25; 398 Tage).

Derselbe Fall wie Abb. 13. Polymorphzelliges Sarkom. Sarkom zellen mit Protoplasmafortsätzen miteinander in Verbindung.

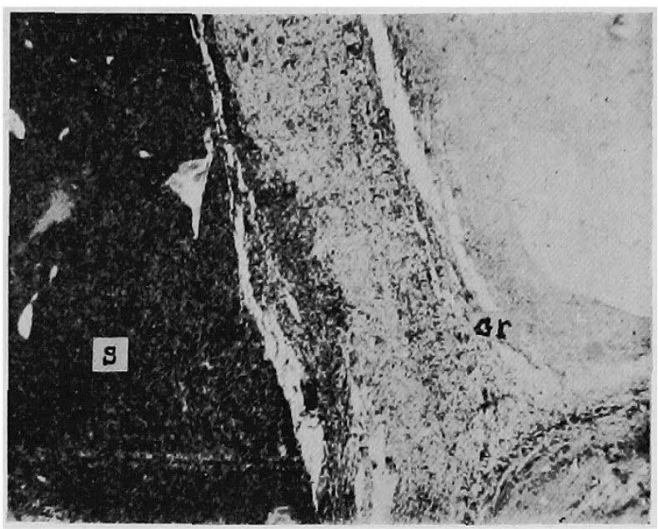

Abb. 20. (Nr. II-16; 476 Tage).

Mikroskopisches Bild des Falls von Abb. 3. Kernarmes Kieselgurgranulom (Gr), Fremdkörperriesenzellen enthaltende Randzone und darauf liegendes Spindelzellensarkom (S).

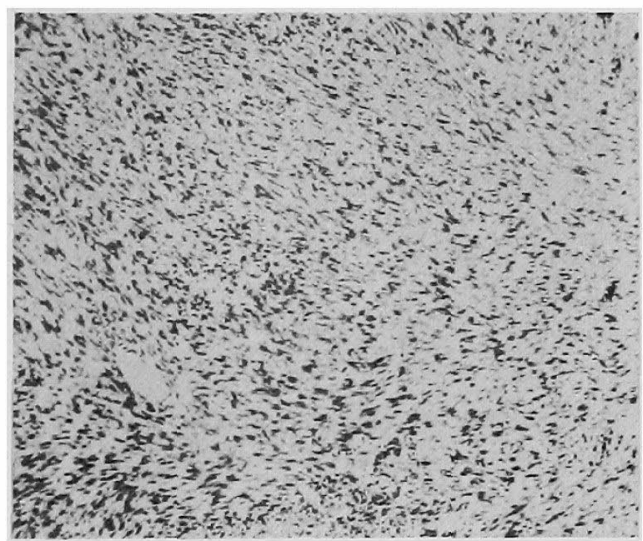

Abb. 22. (Nr. II-16 ; 476 Tage).

Derselbe Fall wie Abb. 20. Mittelstark vergrössert. Spindelzellensarkom. 